\title{
Cerebrospinal fluid amyloid beta and tau proteins in atypical Parkinsonism: a review
}

\author{
Vasilios C. Constantinides ${ }^{1}$, George P. Paraskevas ${ }^{1}$, Fotini Boufidou¹, Mara Bourbouli², Panagiotis G. \\ Paraskevas $^{3}$, Leonidas Stefanis ${ }^{1}$, Elisabeth Kapaki ${ }^{1}$ \\ ${ }^{1} 1$ st Department of Neurology, National and Kapodistrian University of Athens, Athens, P.C. 11528, Greece. \\ 2Department of Neurology, University of Crete, Heraklion 70013, Greece. \\ ${ }^{3}$ Department of Nursing, Technological Educational Institute of Crete, Heraklion 71500, Greece.
}

Correspondence to: Dr. Vasilios C. Constantinides, 1st Department of Neurology, National and Kapodistrian University of Athens, School of Medicine, Eginition Hospital,72 Vas. Sofias Ave, Athens 11528, Greece.

E-mail:vconstan@med.uoa.gr; vassilis.kon@hotmail.com

How to cite this article: Constantinides VC, Paraskevas GP, Boufidou F, Bourbouli M, Paraskevas PG, Stefanis L, Kapaki E. Cerebrospinal fluid amyloid beta and tau proteins in atypical Parkinsonism: a review. Neuroimmuno/ Neuroinflammation 2020;7:120-31. http://dx.doi.org/10.20517/2347-8659.2019.22

Received: 17 Dec 2019 First Decision: 2 Mar 2020 Revised: 25 Mar 2020 Accepted: 7 Apr 2020 Available online: 16 May 2020

Science Editor: Athanassios P. Kyritsis Copy Editor: Jing-Wen Zhang Production Editor: Jing Yu

\begin{abstract}
Progressive supranuclear palsy, corticobasal degeneration, multiple system atrophy and dementia with Lewy bodies are the most common causes of atypical Parkinsonism and enter the differential diagnosis of Parkinson's disease. multiple system atrophy, dementia with Lewy bodies and Parkinson's disease are synucleinopathies, whereas progressive supranuclear palsy and corticobasal degeneration are tauopathies. Multiple cerebrospinal fluid markers have been applied on cohorts of patients with Parkinsonism, with the aim to develop biomarkers for these disorders. Total tau $\left(\tau_{\mathrm{T}}\right)$, phosphorylated tau at threonine $181\left(\tau_{\mathrm{p}}-181\right)$ and amyloid-beta with 42 amino acids $\left(A \beta_{42}\right)$ are considered classical biomarkers for Alzheimer's disease. The aim of the present study is to review the literature regarding these classical cerebrospinal fluid biomarkers in cohorts with Parkinsonism, as well as present data on novel approaches regarding analysis of these proteins.
\end{abstract}

Keywords: Biomarkers, cerebrospinal fluid, progressive supranuclear palsy, corticobasal degeneration, multiple system atrophy, dementia with Lewy bodies, tau protein, phosphorylated tau protein, amyloid beta

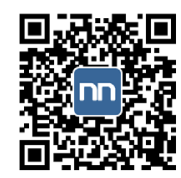




\section{INTRODUCTION}

Parkinson's disease (PD) is the most common neurodegenerative movement disorder. The pathologic hallmarks of PD are Lewy bodies, which consist of intraneuronal cytoplasmic depositions of pathological $\alpha$-synuclein ${ }^{[1]}$. Thus, PD is considered a synucleinopathy. Clinical diagnosis of PD is straightforward in typical cases. However, it can be problematic in patients with atypical clinical features. Accuracy of clinical diagnosis of PD is suboptimal, since as many as $25 \%$ of patients can be misdiagnosed ${ }^{[2]}$.

Progressive supranuclear palsy (PSP), corticobasal degeneration (CBD), multiple system atrophy (MSA) and dementia with Lewy bodies (DLB) are the most common causes of atypical Parkinsonism. All of these diseases manifest with Parkinsonism, which is poorly or only transiently responsive to dopaminergic treatment.

MSA is a synucleinopathy, like PD. Its pathologic hallmark is glial cytoplasmic inclusions, which consist of abnormal $\alpha$-synuclein deposition in oligodendrocytes ${ }^{[3]}$. DLB is also a synucleinopathy, characterized by predominantly cortical Lewy bodies ${ }^{[4]}$. PSP and CBD on the other hand are considered tauopathies, since their main pathologic findings (tufted astrocytes and astrocytic plaques, respectively) consist of abnormal tau protein aggregates in astrocytes ${ }^{[5,6]}$.

Tau protein can present in six isoforms, depending on the alternate splicing of the microtubule associated protein tau $(M A P T)$ gene $^{[7]}$. This results in the variable expression of no, one or two oligonucleotides (N1 and N2) coded by exons 2 and 3 of the MAPT gene, as well as the presence of 3-repeat (3R) or 4-repeat $(4 \mathrm{R})$ microtubule binding regions coded by exon 10 . Depending on the predominance of $3 \mathrm{R}$ - or $4 \mathrm{R}$-tau isoforms, tauopathies are further divided into $4 \mathrm{R}$ - or $3 \mathrm{R}$-tauopahies. PSP and CBD are considered 4-repeat $(4 \mathrm{R})$ tauopathies, Alzheimer's disease $(\mathrm{AD})$ is a mixed $3 \mathrm{R}$ - and $4 \mathrm{R}$-tauopathy, whereas Pick's disease is a 3R-tauopathy.

Total tau protein $\left(\tau_{\mathrm{T}}\right)$, phosphorylated tau protein at threonine $181\left(\tau_{\mathrm{P}-181}\right)$ and amyloid beta with 42 amino acids $\left(A \beta_{42}\right)$ are well-characterized cerebrospinal fluid (CSF) biomarkers of AD. These biomarkers have been incorporated into the most recent $\mathrm{AD}$ diagnostic criteria ${ }^{[8]}$ and are the basis of the recently proposed $\mathrm{AT}(\mathrm{N})$ taxonomy system, which introduces biomarkers (according to their molecular specificity) for the in vivo pathological characterization of patients with $\mathrm{AD}^{[9]}$.

The aim of this review is to present data on the utility of these three classical CSF biomarkers $\left(\tau_{\mathrm{T}}, \tau_{\mathrm{P}-181}\right.$ and $A \beta_{42}$ ) in the differential diagnosis of atypical Parkinsonism from PD. To this end, only studies which included patients with atypical Parkinsonism vs. PD or healthy controls are included.

This review includes representative studies which either have established our current knowledge on CSF biomarkers in Parkinsonism or provide new insights on the subject [Table 1].

\section{STUDIES IN PSP}

Most studies do not report any differences in CSF A $\beta_{42}$ between PSP and other causes of Parkinsonism or controls ${ }^{[10-16]}$. Interestingly, however, some studies have reported lower $A \beta_{42}$ values in PSP compared to controls ${ }^{[17-19]}$. Moreover, a single study reported lower $A \beta_{42}$ levels in PSP $v s . \mathrm{PD}^{[19]}$. According to this study, $\mathrm{A} \beta_{42}$ could differentiate PSP from PD with $83 \%$ sensitivity and $67 \%$ specificity.

Regarding CSF $\tau_{\mathrm{T}}$, several studies could not establish any differences between PSP patients and controls ${ }^{[13-17]}$. Likewise, no difference in $\tau_{\mathrm{T}}$ was evident between PSP and $\mathrm{CBD}^{[13,15-17]}, \mathrm{MSA}^{[13,15,16]}$ or $\mathrm{PD}^{[15,16]}$ in most studies. Two studies have supported that PSP patients exhibit lower $\tau_{\mathrm{T}}$ levels when compared to 
Table 1. Overview of the studies with data on classical CSF biomarkers in cohorts with Parkinsonism

\begin{tabular}{|c|c|c|c|c|}
\hline & $\mathbf{A} \beta_{42}$ & $\tau_{\mathrm{T}}$ & $\tau_{\text {P-181 }}$ & Comparison group \\
\hline \multicolumn{5}{|l|}{ Studies in PSP } \\
\hline Holmberg et al..$^{[10]}$ & $\leftrightarrow$ & & & Ctrl, MSA, PD \\
\hline Verbeek et $a l^{[11]}$ & $\leftrightarrow$ & & & Ctrl, MSA, PD \\
\hline Aerts et al. ${ }^{[12]}$ & $\leftrightarrow^{\mathrm{a}}$ & $\downarrow^{b} \uparrow^{c}$ & $\leftrightarrow^{a}$ & $\begin{array}{l}\text { a. CBD, PD, ctrl } \\
\text { b. } C B D \\
\text { c. } P D\end{array}$ \\
\hline Hall et al. ${ }^{[13]}$ & $\leftrightarrow^{a} \uparrow^{b}$ & $\leftrightarrow^{c}$ & $\downarrow^{d}$ & $\begin{array}{l}\text { a. MSA, CBD, PD, PDD, ctrl } \\
\text { b. DLB } \\
\text { c. ctrl, CBD, MSA } \\
\text { d. ctrl }\end{array}$ \\
\hline Schoonenboom et al. ${ }^{[14]}$ & $\leftrightarrow$ & $\leftrightarrow$ & $\leftrightarrow$ & $\mathrm{DLB}, \mathrm{CBD}, \mathrm{ctrl}$ \\
\hline Magdalinou et al. ${ }^{[15]}$ & $\leftrightarrow$ & $\leftrightarrow$ & $\leftrightarrow$ & CBD, MSA, PD, ctrl \\
\hline Constantinides et $a / .^{[16]}$ & $\leftrightarrow$ & $\leftrightarrow$ & $\leftrightarrow$ & MSA, PD, CBD, ctrl \\
\hline Noguchi et al. ${ }^{[17]}$ & $\downarrow^{\mathrm{a}}$ & $\leftrightarrow^{\mathrm{b}}$ & $\leftrightarrow^{b}$ & $\begin{array}{l}\text { a. ctrl } \\
\text { b. ctrl, CBD }\end{array}$ \\
\hline Wagshal et $a l_{.}^{[18]}$ & $\downarrow$ & $\downarrow$ & $\leftrightarrow$ & ctrl \\
\hline Schirinzi et $a{ }^{\left[{ }^{[19]}\right.}$ & $\downarrow$ & $\downarrow$ & $\downarrow$ & ctrl \\
\hline Süssmuth et al. ${ }^{[20]}$ & $\leftrightarrow$ & $\leftrightarrow$ & $\leftrightarrow$ & MSA, PD \\
\hline \multicolumn{5}{|l|}{ Studies in CBD } \\
\hline Aerts et al. ${ }^{[12]}$ & $\leftrightarrow^{\mathrm{a}}$ & $\uparrow^{a}$ & $\begin{array}{l}\uparrow^{b} \\
\leftrightarrow^{c}\end{array}$ & $\begin{array}{l}\text { a. ctrl, PSP, PD } \\
\text { b. PD } \\
\text { c. ctrl, PSP }\end{array}$ \\
\hline Constantinides et al. ${ }^{[16]}$ & $\downarrow^{\mathrm{a}}$ & $\uparrow^{b} \leftrightarrow^{c}$ & $\leftrightarrow^{d}$ & $\begin{array}{l}\text { a. PD } \\
\text { b. ctrl } \\
\text { c. PSP, MSA, PD } \\
\text { d. PSP, MSA, PD, ctrl }\end{array}$ \\
\hline Mitani et al. ${ }^{[21]}$ & & $\uparrow$ & & ctrl \\
\hline Urakami et al. ${ }^{[22]}$ & & $\uparrow$ & & ctrl, PSP \\
\hline Urakami et al. ${ }^{[23]}$ & & $\uparrow$ & & ctrl, PSP \\
\hline Hall et $a{ }^{[[13]}$ & $\leftrightarrow^{a} \uparrow^{b}$ & $\uparrow^{c} \leftrightarrow^{d}$ & $\leftrightarrow^{e} \uparrow^{\dagger}$ & $\begin{array}{l}\text { a. MSA, PSP, PD, PDD, ctrl } \\
\text { b. DLB } \\
\text { c. PD } \\
\text { d. PSP } \\
\text { e. PSP, PD, ctrl } \\
\text { f. MSA }\end{array}$ \\
\hline Schoonenboom et al. ${ }^{[14]}$ & $\downarrow$ & $\leftrightarrow$ & $\leftrightarrow$ & ctrl \\
\hline Magdalinou et al. ${ }^{[15]}$ & $\leftrightarrow$ & $\leftrightarrow$ & $\leftrightarrow$ & MSA, PSP, PD, ctrl \\
\hline Noguchi et al. ${ }^{[17]}$ & $\leftrightarrow^{\mathrm{a}} \downarrow^{\mathrm{b}}$ & $\leftrightarrow^{c}$ & $\leftrightarrow^{c}$ & $\begin{array}{l}\text { a. PSP } \\
\text { b. ctrl } \\
\text { c. ctrl, PSP }\end{array}$ \\
\hline \multicolumn{5}{|l|}{ Studies in MSA } \\
\hline Holmberg et $a .^{[10]}$ & $\downarrow$ & & & PD, PSP, ctrl \\
\hline Shi et $\left.a\right|^{[26]}$ & $\downarrow^{\mathrm{a}} \leftrightarrow^{\mathrm{b}}$ & $\leftrightarrow^{c}$ & $\leftrightarrow^{c}$ & $\begin{array}{l}\text { a. ctrl } \\
\text { b. PD } \\
\text { c. ctrl,PD }\end{array}$ \\
\hline Verbeek et al. ${ }^{[11]}$ & $\leftrightarrow$ & & & Ctrl, PD \\
\hline Hall et al. ${ }^{[13]}$ & $\leftrightarrow^{\mathrm{a}} \uparrow^{\mathrm{b}}$ & $\uparrow^{c}$ & $\downarrow^{d}$ & $\begin{array}{l}\text { a. MSA, CBD, PD, PDD, ctrl } \\
\text { b. DLB } \\
\text { c. PD, PDD } \\
\text { d. PD, ctrl }\end{array}$ \\
\hline Magdalinou et al. ${ }^{[15]}$ & $\leftrightarrow$ & $\leftrightarrow$ & $\leftrightarrow$ & CBD, PSP, PD, ctrl \\
\hline Constantinides et $a{ }^{[16]}$ & $\leftrightarrow$ & $\leftrightarrow$ & $\leftrightarrow$ & PSP, CBD, PD, ctrl \\
\hline Süssmuth et $a{ }^{[20]}$ & $\leftrightarrow^{\mathrm{a}}$ & $\uparrow^{b}$ & $\leftrightarrow^{c}$ & $\begin{array}{l}\text { a. PD, PSP } \\
\text { b. ctrl, PD } \\
\text { c. PSP, PD, ctrl }\end{array}$ \\
\hline Mollenhauer et al. ${ }^{[27]}$ & $\leftrightarrow$ & $\leftrightarrow$ & & PSP, PD \\
\hline Abdo et al. ${ }^{[28]}$ & & $\uparrow$ & & ctrl \\
\hline Herbert et al. ${ }^{[29]}$ & & $\uparrow$ & & Ctrl, PD \\
\hline Abdo et al. ${ }^{[30]}$ & & $\uparrow$ & & PD \\
\hline Herbert et $a{ }^{[31]}$ & & $\uparrow$ & & PD \\
\hline \multicolumn{5}{|l|}{ Studies in DLB } \\
\hline Schoonenboom et al. ${ }^{[14]}$ & $\downarrow^{a} \uparrow^{b}$ & $\downarrow^{b} \uparrow^{a}$ & $\downarrow^{b} \uparrow^{a}$ & $\begin{array}{l}\text { a. ctrl } \\
\text { b. AD }\end{array}$ \\
\hline
\end{tabular}




\begin{tabular}{|c|c|c|c|c|}
\hline Mollenhauer et al.. ${ }^{[32]}$ & $\downarrow^{\mathrm{a}} \leftrightarrow^{\mathrm{b}}$ & $\downarrow^{b} \leftrightarrow^{a}$ & $\uparrow^{a}$ & $\begin{array}{l}\text { a. ctrl } \\
\text { b. } A D\end{array}$ \\
\hline Mollenhauer et al.. ${ }^{[33]}$ & $\downarrow^{a} \leftrightarrow^{b}$ & $\downarrow^{b} \uparrow^{a}$ & $\leftrightarrow^{\mathrm{b}}$ & $\begin{array}{l}\text { a. ctrl } \\
\text { b. } A D\end{array}$ \\
\hline Mulugeta et $a .^{[34]}$ & $\downarrow^{a} \leftrightarrow^{b}$ & $\leftrightarrow^{c}$ & $\leftrightarrow^{c}$ & $\begin{array}{l}\text { a. ctrl } \\
\text { b.AD, PDD } \\
\text { c. ctrl, PDD }\end{array}$ \\
\hline Llorens et al. $^{[35]}$ & $\downarrow^{\mathrm{a}}$ & $\uparrow^{b}$ & & $\begin{array}{l}\text { a. ctrl, PD, PDD } \\
\text { b. PD, MSA }\end{array}$ \\
\hline Van Steenoven et al. ${ }^{[36]}$ & $\downarrow$ & & & ctrl \\
\hline Parnetti et al.. ${ }^{[37]}$ & $\downarrow^{a} \leftrightarrow \leftrightarrow^{b}$ & $\downarrow^{b} \uparrow^{c}$ & $\downarrow^{b}$ & $\begin{array}{l}\text { a. PD, PDD } \\
\text { b. AD } \\
\text { c. PD }\end{array}$ \\
\hline Kaerst et al. ${ }^{[38]}$ & $\downarrow$ & $\uparrow$ & & PD \\
\hline Gmitterova et al. ${ }^{[39]}$ & $\downarrow$ & $\uparrow$ & & PDD \\
\hline Gómez-Tortosa et al. ${ }^{[40]}$ & $\uparrow^{a} \leftrightarrow^{b}$ & $\downarrow^{\mathrm{a}} \leftrightarrow^{\mathrm{b}}$ & & $\begin{array}{l}\text { a. } A D \\
\text { b. ctrl }\end{array}$ \\
\hline Boström et al. ${ }^{[41]}$ & $\uparrow^{a}$ & $\downarrow^{\mathrm{a}}$ & $\uparrow^{b}$ & $\begin{array}{l}\text { a. } A D \\
\text { b. ctrl }\end{array}$ \\
\hline Abdelnour et al. ${ }^{[42]}$ & $\uparrow$ & & & $\operatorname{ctrl}$ \\
\hline Mollenhauer et al. ${ }^{[43]}$ & & $\leftrightarrow$ & & PDD \\
\hline
\end{tabular}

个: elevated CSF levels; $\downarrow$ : decreased CSF levels; $\leftrightarrow$ : no difference in CSF levels; CSF: cerebrospinal fluid; AD: Alzheimer's disease; PD: Parkinson's disease; MSA: multiple system atrophy; PSP: progressive supranuclear palsy; CBD: corticobasal degeneration; DLB: dementia with Lewy bodies; PDD: parkinson's disease dementia

controls $^{[18,19]}$, whereas a single study posited that patients with PSP have lower $\tau_{\mathrm{T}}$ levels compared to CBD, but higher $\tau_{\mathrm{T}}$ levels compared to $\mathrm{PD}^{[12]}$.

CSF $\tau_{\text {P-181 }}$ does not seem to be useful in the differentiation of PSP from other causes of Parkinsonism or controls $^{[12,14-18]}$. Two studies have reported lower $\tau_{\mathrm{P}-181}$ levels compared to controls ${ }^{[13,19]}$.

Few studies include data on ratios of CSF biomarkers. In a large study, which included PSP, CBD and $\mathrm{AD}$ patients, elevated $\tau_{\mathrm{P}-181} / \mathrm{A} \beta_{42}$ ratio values could differentiate AD from PSP and $\mathrm{CBD}^{[17]}$. Another study posited that lower $\tau_{\mathrm{P}-181} / \tau_{\mathrm{T}}$ ratio values could discriminate patients with atypical Parkinsonism (PSP and MSA) from $\mathrm{PD}^{[20]}$. This ratio was optimal for discriminating PSP from CBD with a reported sensitivity of $86 \%$ and specificity of $75 \%{ }^{[12]}$. PSP patients could also be differentiated from controls by lower $\mathrm{A} \beta_{42} / \tau_{\mathrm{T}}^{[12]}$ and higher $\tau_{\mathrm{T}} / \tau_{\mathrm{P}-181}{ }^{[16]}$ ratios.

A single study examined classical CSF biomarkers in different phenotypes of PSP. To this end, patients with classic Richardson's syndrome (RS) were compared to patients with PSP-Parkinsonism (PSP-P). Interestingly, only patients with PSP-P had elevated total $\tau_{\mathrm{T}}$ levels, compared to RS, PD and controls. A $\beta_{42}$ on the other hand was significantly lower in RS patients compared to PSP-P ${ }^{[20]}$.

Few PSP patients seem to harbor a CSF-AD profile (generally defined as decreased $\mathrm{A} \beta_{42}$ with elevated $\tau_{\mathrm{T}}$ or $\left.\tau_{\mathrm{P}-181}\right)$. In a large cohort, including diverse neurodegenerative disorders, $10 \%$ of PSP patients had a CSF$\mathrm{AD}$ profile, as determined by an index incorporating CSF $\mathrm{A} \beta_{42}$ and $\tau_{\mathrm{P}-181}$ values ${ }^{[14]}$. Likewise, only one of 19 PSP patients $(\sim 5 \%)$ had a typical CSF-AD profile, as determined by abnormal $\mathrm{A} \beta_{42}, \tau_{\mathrm{T}}$ and $\tau_{\mathrm{P}-181}$ values, in a cohort of patients with Parkinsonism ${ }^{[16]}$.

Lastly, the possible relationship between CSF biomarkers and clinical characteristics has also been extensively studied in PSP. Most studies agree that there is no clinical-biochemical correlation in PSP ${ }^{[12-15,17]}$. Two studies have correlated low $A \beta_{42}$ levels with higher disease severity, as measured by Hoehn and Yahr score $^{[10]}$ or the PSP Rating Scale ${ }^{[19]}$. 
As a general conclusion, there are no significant abnormalities in the classical CSF biomarkers in PSP. Despite being a 4R-tauopathy, CSF tau proteins do not seem to differ between PSP and other Parkinsonian disorders. CSF total tau protein, which is considered a non-specific marker of neurodegeneration, would be expected to increase in PSP, as is the case in AD. A plausible explanation would be that tau protein in PSP is concentrated intracellularly (in astrocytic plaques, tufted astrocytes and neurofibrillary tangles), and for this reason it does not enter the cerebrospinal fluid.

Additionally, no obvious clinical-biochemical correlation has been observed, while an AD biomarker profile in PSP (indicating an AD co-occurrence or possibly a PSP-like phenotype of AD) is a rarity. The presence of a CSF-AD profile in PSP patients is more likely to be indicative of the presence of dual underlying pathologies (AD and PSP), since pathological studies have not established a correlation of $\mathrm{AD}$ pathology and Richardson syndrome (the most common phenotype of PSP).

\section{STUDIES IN CBD}

Most studies have focused on CSF tau proteins in CBD, since CBD is a tauopathy. Several of these studies have reported elevated levels of $\tau_{\mathrm{T}}$ in $\mathrm{CBD}$, compared to controls ${ }^{[12,16,21-23]}, \mathrm{PSP}^{[12,22,23]}$ and $\mathrm{PD}^{[12,13]}$. Interestingly, $\tau_{\mathrm{T}}$ could differentiate CBD from PSP with $80 \%$ specificity and sensitivity in one study ${ }^{[23]}$. Likewise, CSF $\tau_{\mathrm{T}}$ provided $75 \%$ sensitivity and $90 \%$ specificity in the discrimination of CBD from PD in another study ${ }^{[12]}$. However, no difference in $\tau_{\mathrm{T}}$ between $\mathrm{CBD}$ and controls ${ }^{[14,15,17]}, \mathrm{PSP}^{[13,15,16,20]}, \mathrm{MSA}^{[15,16]}$ and $\mathrm{PD}^{[15,16]}$ has also been reported.

Regarding CSF $\tau_{\mathrm{P}-181}$, most studies did not establish any meaningful difference between CBD patients and other Parkinsonian disorders or controls ${ }^{[13-17]}$. A single study has reported elevated CSF $\tau_{\text {P-181 }}$ levels in CBD compared to $\mathrm{PD}^{[12]}$, and another study in CBD patients compared to MSA patients ${ }^{[13]}$.

CSF $A \beta_{42}$ levels do not differ in CBD compared to other Parkinsonian disorders, according to several studies $^{[12,13,15,17]}$. However, lower $A \beta_{42}$ has been described in CBD patients compared to controls ${ }^{[14,17]}$ and $\mathrm{PD}^{[16]}$.

Regarding CSF biomarker indices, few studies have included relevant data in CBD. One study reported decreased $\mathrm{A} \beta_{42} / \tau_{\mathrm{T}}$ ratio in CBS compared to PD and controls, whereas $\tau_{\mathrm{P}-181} / \tau_{\mathrm{T}}$ ratio in CBS was decreased compared to controls ${ }^{[12]}$. According to this study, $\tau_{\mathrm{P}-181} / \tau_{\mathrm{T}}$ was optimal for PSP $v$ s. CBD discrimination (86\% sensitivity and $75 \%$ specificity). Another study posited that CBD patients have elevated $\tau_{\mathrm{T}} / \mathrm{A} \beta_{42}$ and $\tau_{\mathrm{P}-181} /$ $\mathrm{A} \beta_{42}$ compared to $\mathrm{PD}^{[16]}$.

Clinical and demographic data do not correlate with levels of classical CSF biomarkers in CBD, according to most relevant studies ${ }^{[13-15]}$. A single study reported that cognitive status, as measured by MMSE, correlated with both $\tau_{\mathrm{T}}$ and $\tau_{\mathrm{P}-181}$ levels and was inversely correlated with $\mathrm{A} \beta_{42}$ levels ${ }^{[12]}$. Intriguingly, $\tau_{\mathrm{T}}$ levels in CBD and PSP were dependent on disease severity in a single study, with maximal levels in medium-stage disease ${ }^{[23]}$.

Few studies have focused on the presence of a CSF-AD profile in cohorts of Parkinsonian disorders. According to this approach, patients are divided in those harboring a CSF-AD profile and those who do not harbor such a profile. An initial study concluded that $20 \%$ of CBS patients harbored a CSF-AD profile (as defined by $\tau_{\mathrm{T}}>400 \mathrm{pg} / \mathrm{mL}, \mathrm{A} \beta_{42}<400 \mathrm{pg} / \mathrm{mL}$ and $\tau_{\mathrm{T}} / \mathrm{A} \beta_{42}$ ratio $\left.>1\right)^{[24]}$. In another cohort, $38 \%$ of CBS patients had a CSF-AD profile, based on an index which included $\tau_{\text {P-181 }}$ and $A \beta_{42}$ values ${ }^{[14]}$. Along the same lines, a third study posited that $\sim 30 \%$ of CBS patients had a typical CSF-AD profile (elevated $\tau_{\mathrm{T}}$ and $\tau_{\mathrm{P}-181}$ combined with decreased $\left.A \beta_{42}\right)^{[16]}$. Interestingly, when patients with a CSF-AD profile were excluded from analyses, an initially reported elevated $\tau_{\mathrm{T}}$ and decreased $\mathrm{A} \beta_{42}$ protein in CBS disappeared, and CBS patients 
no longer differed from PD or controls. This implied that the elevated $\tau_{\mathrm{T}}$ and decreased $\mathrm{A} \beta_{42}$ levels in CBS might have been the result of the inclusion of AD patients who presented with a CBS phenotype. The CSF profile was implemented in another study by the same study group to investigate possible differences between $\mathrm{AD}$ and non-AD pathology in a CBS cohort $^{[25]}$.

In conclusion, abnormalities in classical CSF biomarkers are common in CBD. The commonly reported elevation in $\tau_{\mathrm{T}}$ and $\tau_{\mathrm{P}-181}$ and decrease in $\mathrm{A} \beta_{42}$, does not seem to be a biochemical fingerprint of CBD but rather is a result of the inclusion in analyses of patients with AD pathology and a CBS phenotype. Up to $30 \%$ of CBS patients have an AD-biomarker profile, which is in accordance with pathological studies. Thus, CSF biomarkers are particularly useful tools in the in vivo discrimination of corticobasal syndrome in CBSnon-AD and CBS-AD.

\section{STUDIES IN MSA}

An initial study on CSF A $\beta_{42}$ in MSA reported lower levels compared to PD, PSP and controls ${ }^{[10]}$. A different study group also found lower $A \beta_{42}$ levels in MSA compared to controls ${ }^{[26]}$. However, several other studies could not establish any meaningful difference in $A \beta_{42}$ levels in MSA compared to other Parkinsonian disorders or controls ${ }^{[11,13,15,16,20,26,27]}$.

Results regarding $\tau_{\mathrm{T}}$ in MSA are conflicting, with most studies reporting an increase of $\tau_{\mathrm{T}}$ in MSA compared to either controls ${ }^{[20,28,29]}$ or other Parkinsonian disorders ${ }^{[13,20,29-31]}$. However, some studies have reported no difference between MSA and other Parkinsonian disorders ${ }^{[16,26,27]}$, whereas a single study has supported that MSA patients exhibit lower $\tau_{\mathrm{T}}$ compared to controls ${ }^{[26]}$.

Few studies have included $\tau_{\mathrm{P}-181}$ data in the comparison of biomarkers in MSA and other Parkinsonian disorders. These studies did not find any difference in $\tau_{\mathrm{P}-181}$ levels in MSA compared to other study groups ${ }^{[15,16,20,26]}$. A single study has supported lower $\tau_{\mathrm{P}-181}$ levels in MSA compared to PD and controls ${ }^{[13]}$.

Two studies have included CSF biomarker indices in the differential diagnosis of MSA from related disorders. More specifically, a study has supported that MSA patients have significantly lower $\tau_{\mathrm{P}-181} / \tau_{\mathrm{T}}$ ratios compared to $\mathrm{PD}^{[20]}$. Another study posited that higher values of $\tau_{\mathrm{T}} / \mathrm{A} \beta_{42}$ ratio could differentiate MSA from PD with high specificity but only moderate sensitivity ${ }^{[16]}$.

Regarding disease subtypes, MSA-C and MSA-P patients did not differ in their CSF biochemical profile in all relevant studies ${ }^{[1,20,28,30]}$.

Clinical-biochemical correlation studies in MSA are sparse. A study correlated disease severity in MSA with lower CSF $A \beta_{42}$ levels ${ }^{[10]}$. Another study supported that both $\tau_{\mathrm{T}}$ and $\tau_{\mathrm{P}-181}$ levels increased with age in $\mathrm{MSA}^{[26]}$. A study implementing a battery of CSF biomarkers in a cohort of diverse neurodegenerative disorders could not establish any correlation between CSF biomarkers and clinical characteristics ${ }^{[15]}$. In conclusion, data on classical CSF biomarkers are largely inconclusive.

\section{STUDIES IN DLB}

It is well documented that CSF $A \beta_{42}$ in DLB is decreased compared to controls ${ }^{[14,32-36]}, \mathrm{PD}^{[35,37,38]}$ and parkinson's disease dementia (PDD) ${ }^{[5,3,37,39]}$ according to the majority of studies on the subject. Comparison of $A \beta_{42}$ levels between $A D$ and DLB has yielded conflicting results, with some studies reporting greater $A \beta_{42}$ levels in DLB compared to $\mathrm{AD}^{[14,40,41]}$, and other studies not reporting any significant difference between the two groups ${ }^{[32-34,37]}$. A single study reported greater $A \beta_{42}$ values in DLB compared to controls ${ }^{[42]}$ and another study did not find any difference between the two groups ${ }^{[40]}$. Another study did not report a difference between DLB and PDD ${ }^{[34]}$. 
There is consensus among studies that CSF $\tau_{\mathrm{T}}$ is increased in AD compared to DLB ${ }^{[14,32,33,37,40,41]}$. DLB patients have been reported to harbor elevated $\tau_{\mathrm{T}}$ levels compared to controls ${ }^{[1,33]}$, although several studies could not establish any difference between DLB and controls ${ }^{[32,34,40]}$. Regarding other synucleinopathies (PD, PDD and MSA), studies have produced mixed results, reporting either an increase in $\tau_{\mathrm{T}}$ in DLB compared to $\mathrm{PD} / \mathrm{PDD}^{[35,37-39]}$ and $\mathrm{MSA}^{[35]}$ or no difference among patient groups ${ }^{[34,43]}$.

Few studies have included $\tau_{\mathrm{P}-181}$ in analyses of DLB cohorts. According to these limited studies, $\tau_{\mathrm{P}-181}$ is elevated in $\mathrm{AD}$ compared to $\mathrm{DLB}^{[14,37]}$, although one study could not establish a difference between $\mathrm{AD}$ and $\mathrm{DLB}^{[33]}$. Comparison between DLB and controls regarding $\tau_{\mathrm{P}-181}$ has produced mixed results, with some studies reporting an increase in $\tau_{\mathrm{P}-181}$ in $\mathrm{DLB}^{[14,32,41]}$, whereas other studies could not establish any difference between groups ${ }^{[34]}$. PD/PDD patients do not differ from DLB in their $\tau_{\mathrm{P}-181}$ profile ${ }^{[34,44]}$.

Lower CSF A $\beta_{42}$ levels in DLB correlate with a worse outcome, according to several studies ${ }^{[14,40,42,45]}$. Likewise, elevated $\tau_{\mathrm{T}}$ levels correlate with a poorer prognosis in $\mathrm{DLB}^{[37-39,41]}$.

Several studies in DLB, which contain longitudinal data regarding CSF biomarker level alterations over time, provide conflicting results. Two studies have reported stable $A \beta_{42}$ over time in cohorts of DLB patients ${ }^{[39,46]}$. According to these studies, CSF $\tau_{\mathrm{T}}$ and $\tau_{\mathrm{P}-181}$ levels either increase ${ }^{[39]}$ or decrease over time ${ }^{[46]}$. Conversely, two other studies have provided a different profile, with unaltered $\tau_{\mathrm{T}}$ and $\tau_{\mathrm{P}-181}$ levels over time, combined with a decrease in $A \beta_{42}$ during the transition from prodromal to demented stage of DLB ${ }^{[47,48]}$.

A CSF-AD profile (as defined by a decrease in $A \beta_{42}$ and an increase in $\tau_{\mathrm{T}}$ and/or $\tau_{\mathrm{P}-181}$ ) is common in DLB patients, presenting in $25 \%-50 \%$ of patients ${ }^{[14,42,45,49]}$. This profile invariably correlates with poorer prognosis ${ }^{[42,45}$. According to a recent study, as many as $85 \%$ of DLB patients have decreased $A \beta_{42}$ in CSF, either isolated $(45 \%)$ or in the context of a CSF-AD profile $(40 \%)^{[49]}$.

A single study has provided insight into the relationship between pathological finding and CSF biochemical profile ${ }^{[4]]}$. In this cohort, $72 \%$ of DLB patients had senile plaques and $50 \%$ had neurofibrillary tangles. Interestingly, there was a correlation between the presence of senile plaques and CSF A $\beta_{42}$ levels. The cooccurrence of neurofibrillary tangles did not affect $\tau_{\mathrm{T}}$ and $\tau_{\mathrm{P}-181}$ levels in DLB patients.

In conclusion, there is a general agreement that $A \beta_{42}$ levels are decreased in DLB, either alone or accompanied by increased $\tau_{\mathrm{T}}$ or $\tau_{\mathrm{P}-181}$ levels. The decrease of $\mathrm{A} \beta_{42}$ levels have been shown to correlate with faster disease progression. Decreased CSF A $\beta_{42}$ levels and increased $\tau_{\mathrm{T}}$ and $\tau_{\mathrm{P}-181}$ in DLB could be attributed to the frequent co-occurrence of AD pathology in patients with Lewy body disease. This hypothesis would explain the correlation of a CSF-AD profile with worse outcomes in DLB patients. Intriguingly, multiple lines of evidence suggest an interplay between $\alpha$-synuclein and tau/amyloid beta aggregation, which may explain the frequent co-occurrence of AD pathology and Lewy body disease ${ }^{[50-52]}$.

\section{METHODOLOGICAL CONSIDERATIONS}

Most studies on classical biomarkers in atypical Parkinsonism implemented enzyme-linked immunosorbent assays (ELISAs). Advantages of ELISAs include relatively low cost, high reproducibility and high availability, since commercial ELISAs of classical CSF biomarkers are readily available. There have been efforts, however, to implement novel techniques and to target different proteins in search of new biomarkers in neurodegenerative diseases.

To this end, a study examined differences in tau isoforms in CSF, which may reflect differences in posttranslational processing of tau protein. More specifically, differences in tau proteolytic products in CSF were the main study endpoint. By means of immunoprecipitation, extended ( $55 \mathrm{kDa})$ and truncated forms 
$(33 \mathrm{kDa})$ of tau protein were determined in CSF. The tau $33 \mathrm{kDa} / 55 \mathrm{kDa}$ ratio was significantly lower in PSP patients, compared to patients with AD, FTD, CBD, PD and DLB and emerged as a possible biomarker for PSP ${ }^{[53]}$. The same study group replicated these results in a larger cohort ${ }^{[54]}$. However, these promising results were not be replicated by another study group ${ }^{[55]}$.

In another study, immuno-PCR essays were developed to measure $3 \mathrm{R}$ - and $4 \mathrm{R}$-tau isoforms in CSF. These essays were tested in four different cohorts, which included PSP, CBD, PDD and PDD patients. The study we are referring to included four cohorts, in which the $3 \mathrm{R}$ and $4 \mathrm{R}$-tau isoform levels varied considerably among the four cohorts (mean values in control groups ranging for both $3 \mathrm{R}$ - and $4 \mathrm{R}$-tau from $<5 \mathrm{pg} / \mathrm{mL}$ to $\sim 50 \mathrm{pg} / \mathrm{mL}$ ). Analysis of tau isoforms in all cohorts combined indicated lower $4 \mathrm{R}$-tau levels in PSP and AD. 3R-tau did not differ among study groups. The $3 \mathrm{R}$-tau $/ \tau_{\mathrm{T}}$ and $3 \mathrm{R}$-tau $/ \tau_{\mathrm{P}-181}$ ratios were decreased in $\mathrm{AD}$ compared to PDD. The $4 \mathrm{R}$-tau $/ \tau_{\mathrm{T}}$ was lower in PSP and AD compared to controls and the $4 \mathrm{R}-\mathrm{tau} / \tau_{\mathrm{P}-181}$ ratio was higher in PDD compared to PSP, CBD and $\mathrm{AD}^{[56]}$.

Wagshal et al. ${ }^{[18]}$ developed a multitude of novel ELISAs, which target different epitopes of tau proteins, in order to examine differences in the concentration of diverse tau protein fragments in CSF. These ELISAs were applied in patients with AD, PSP and controls. Interestingly, PSP patients had lower concentrations of most tau fragments compared to controls.

Another study group implemented mass spectrometry to quantify tau-specific peptides in the entire sequence of tau protein in a cohort of PSP, AD and DLB. This novel approach yielded data for 18 tau peptides. By use of these peptides, the authors determined that the $1 \mathrm{~N}$ and $3 \mathrm{R}$-tau isoforms were mostly represented in CSF. Levels of tau peptides were intra-correlated and significantly increased in AD patients. Interestingly, $\mathrm{AD}$ patients had relatively decreased levels of peptides in the central core region of tau protein. This region contains phosphorylation sites, which may explain this finding ${ }^{[57]}$.

Along the same lines, Cicognola et al. ${ }^{[58]}$ developed novel antibodies against tau fragments at amino acid 123 and 224 (tau-123 and tau-224, respectively), after having identified endogenous tau fragments ending at these amino acids. They concluded that anti-tau-224, but not anti-tau-123, was present in neurofibrillary tangles and neuropil threads of AD patients, whereas tau-224 levels were elevated in CSF of these patients and correlated with conversion from mild cognitive impairment to AD. The authors concluded that only tau-224 is neuron-specific, whereas tau-123 may represent a general non-specific marker of tau metabolism.

\section{DISCUSSION}

There has been extensive research on classical CSF biomarkers in patients with Parkinsonism, as illustrated in this review of the literature. Most studies agree that PSP patients do not exhibit a specific CSF biochemical profile. Few studies have reported decreased $A \beta_{42}$ levels. A CSF-AD profile has rarely been described in PSP patients.

An elevation in CSF $\tau_{\mathrm{T}}$ levels has been systematically reported in CBS. Moreover, few studies have also documented a decrease in $A \beta_{42}$ and an increase in $\tau_{\text {P-181 }}$ in these patients. Interestingly, a significant percentage $(<40 \%)$ of CBS patients can harbor a CSF-AD profile ${ }^{[14,16,24]}$. This is in agreement with clinicalpathological studies of $\mathrm{CBS}^{[59-62]}$, where $\mathrm{AD}$ is a relatively common underlying pathology of CBS. A study emphasized that the frequently reported elevation in CSF $\tau_{\mathrm{T}}$ and reduction in $\mathrm{A} \beta_{42}$ in CBS might not represent an inherent biochemical characteristic of CBS and could be attributed to the inclusion of $\mathrm{AD}$ patients presenting with $\mathrm{CBS}^{[16]}$. Thus, classical CSF biomarkers are particularly important in CBS cohorts, since they can indicate an underlying AD pathology.

Regarding MSA, most studies report an elevation in CSF $\tau_{\mathrm{T}}$ protein, whereas a decrease in $A \beta_{42}$ levels in MSA has been rarely reported. Although an interplay between $\alpha$-synuclein and $A \beta_{42}$ has been established 
in vitro, alterations in CSF $A \beta_{42}$ levels in a synucleinopathy are difficult to explain ${ }^{[63]}$. In a transgenic mouse model with expression of both $\beta$-amyloid peptides and synuclein, $\beta$-amyloid peptides promoted aggregation of $\alpha$-synuclein ${ }^{[50]}$. Likewise, pathological data from PD and DLB patients support the accumulation of tau oligomers in these brains. Moreover, oligomeric tau forms may co-exist in the same aggregates with $\alpha$-synuclein, forming hybrid oligomers ${ }^{[51]}$. Along the same lines, $\alpha$-synuclein may induce specific toxic tau oligomers in cell cultures ${ }^{[52]}$.

DLB patients exhibit significantly lower CSF $A \beta_{42}$ values compared to other Parkinsonian disorders. Moreover, studies support that $\tau_{\mathrm{T}}$ in DLB may be elevated. However, AD can be differentiated from DLB by means of a significant increase in $\tau_{\mathrm{T}}$ and $\tau_{\mathrm{P}-181}$ in $\mathrm{AD}$ compared to DLB. A significant percentage of DLB patients harbor a CSF-AD profile, which indicates the frequent co-occurrence of Lewy body and AD pathologies, especially in older patients.

\section{CONCLUSIONS AND FUTURE DIRECTIONS}

Classical CSF biomarkers in atypical Parkinsonism are important, particularly for the diagnostic workup of CBS and DLB patients. A CSF-AD profile in a CBS patient indicates an underlying AD pathology. Conversely, in DLB patients, the presence of a CSF-AD profile indicates the co-occurrence of AD and Lewy body pathologies, which usually correlates with poorer prognosis. Although some differences have been reported in classical CSF biomarkers in both PSP and MSA, the results are inconsistent and require further research.

The disparity of results on classical biomarkers in atypical Parkinsonism can be largely attributed to the great heterogeneity of studies. This heterogeneity refers to diagnostic criteria, cohort synthesis, statistical analysis and pre-analytical and analytical factors. Standardization of these pre-analytical and analytical confounders, as established in recent recommendations, is paramount for more robust results ${ }^{[64]}$. Moreover, large cohorts of Parkinsonian disorders with CSF biomarker data and pathology-confirmed diagnoses are lacking. These studies would reliably inform us on the interplay between CSF biomarkers and pathological findings. This disparity in results also illustrates the limitations of classical biomarkers in the differential diagnosis of patients with Parkinsonism, and emphasizes the need for novel approaches.

To this end, differences in isoforms of these known proteins (e.g., 3R- vs. $4 \mathrm{R}$-tau protein), differences in proteolytic products and quantification of specific peptides of these proteins are promising new approaches. Moreover, better characterization of other proteins which may serve as biomarkers (such as $\alpha$-synuclein or TDP-43) is of great importance.

\section{DECLARATIONS}

\section{Authors' contributions}

Conception of the study, drafting of the manuscript, literature review: Constantinides VC

Conception of the study, critical appraisal of manuscript: Paraskevas GP, Boufidou F

Conception of the study, critical appraisal of manuscript, literature review: Bourbouli M, Paraskevas PG

Conception of the study, critical appraisal of manuscript: Stefanis L, Kapaki E

\section{Availability of data and materials}

Not applicable.

\section{Financial support and sponsorship}

None. 


\section{Conflicts of interest}

All authors declared that there are no conflicts of interest.

\section{Ethical approval and consent to participate}

Not applicable.

\section{Consent for publication}

Not applicable.

\section{Copyright}

(c) The Author(s) 2020.

\section{REFERENCES}

1. Spillantini MG, Schmidt ML, Lee VM, Trojanowski JQ, Jakes R, et al. Alpha-synuclein in Lewy bodies. Nature 1997;388:839-40.

2. Hughes AJ, Daniel SE, Kilford L, Lees AJ. Accuracy of clinical diagnosis of idiopathic Parkinson's disease: a clinico-pathological study of 100 cases. J Neurol Neurosurg Psychiatry 1992;55:181-4.

3. Trojanowski JQ, Revesz T; Neuropathology Working Group on MSA. Proposed neuropathological criteria for the post mortem diagnosis of multiple system atrophy. Neuropathol Appl Neurobiol 2007;33:615-20.

4. McKeith IG, Galasko D, Kosaka K, Perry EK, Dickson DW, et al. Consensus guidelines for the clinical and pathologic diagnosis of dementia with Lewy bodies (DLB): report of the consortium on DLB international workshop. Neurology 1996;47:1113-24.

5. Hauw JJ, Daniel SE, Dickson D, Horoupian DS, Jellinger K, et al. Preliminary NINDS neuropathologic criteria for Steele-RichardsonOlszewski syndrome (progressive supranuclear palsy). Neurology 1994;44:2015-9.

6. Dickson DW, Bergeron C, Chin SS, Duyckaerts C, Horoupian D, et al. Office of rare diseases neuropathologic criteria for corticobasal degeneration. J Neuropathol Exp Neurol 2002;61:935-46.

7. Goedert M, Spillantini MG, Jakes R, Rutherford D, Crowther RA. Multiple isoforms of human microtubule-associated protein tau: sequences and localization in neurofibrillary tangles of Alzheimer's disease. Neuron 1989;3:519-26.

8. McKhann GM, Knopman DS, Chertkow H, Hyman BT, Jack CR Jr, et al. The diagnosis of dementia due to Alzheimer's disease: recommendations from the National Institute on Aging-Alzheimer's Association workgroups on diagnostic guidelines for Alzheimer's disease. Alzheimers Dement 2011;7:263-9.

9. Jack CR Jr, Bennett DA, Blennow K, Carrillo MC, Dunn B, et al. NIA-AA Research Framework: Toward a biological definition of Alzheimer's disease. Alzheimers Dement 2018;14:535-62.

10. Holmberg B, Johnels B, Blennow K, Rosengren L. Cerebrospinal fluid Abeta42 is reduced in multiple system atrophy but normal in Parkinson's disease and progressive supranuclear palsy. Mov Disord 2003;18:186-90.

11. Verbeek MM, Abdo WF, De Jong D, Horstink MW, Kremer BP, et al. Cerebrospinal fluid Abeta42 levels in multiple system atrophy. Mov Disord 2004; 19:238-40.

12. Aerts MB, Esselink RA, Bloem BR, Verbeek MM. Cerebrospinal fluid tau and phosphorylated tau protein are elevated in corticobasal syndrome. Mov Disord 2011;26:169-73.

13. Hall S, Öhrfelt A, Constantinescu R, Andreasson U, Surova Y, et al. Accuracy of a panel of 5 cerebrospinal fluid biomarkers in the differential diagnosis of patients with dementia and/or parkinsonian disorders. Arch Neurol 2012;69:1445-52.

14. Schoonenboom NS, Reesink FE, Verwey NA, Kester MI, Teunissen CE, et al. Cerebrospinal fluid markers for differential dementia diagnosis in a large memory clinic cohort. Neurology 2012;78:47-54.

15. Magdalinou NK, Paterson RW, Schott JM, Fox NC, Mummery C, et al. A panel of nine cerebrospinal fluid biomarkers may identify patients with atypical parkinsonian syndromes. J Neurol Neurosurg Psychiatry 2015;86:1240-7.

16. Constantinides VC, Paraskevas GP, Emmanouilidou E, Petropoulou O, Bougea A, et al. CSF biomarkers beta-amyloid, tau proteins and a-synuclein in the differential diagnosis of Parkinson-plus syndromes. J Neurol Sci 2017;382:91-5.

17. Noguchi M, Yoshita M, Matsumoto Y, Ono K, Iwasa K, et al. Decreased beta-amyloid peptide42 in cerebrospinal fluid of patients with progressive supranuclear palsy and corticobasal degeneration. J Neurol Sci 2005;237:61-5.

18. Wagshal D, Sankaranarayanan S, Guss V, Hall T, Berisha F, et al. Divergent CSF tau alterations in two common tauopathies: Alzheimer's disease and progressive supranuclear palsy. J Neurol Neurosurg Psychiatry 2015;86:244-50.

19. Schirinzi T, Sancesario GM, Di Lazzaro G, Scalise S, Colona VL, et al. Clinical value of CSF amyloid-beta-42 and tau proteins in Progressive Supranuclear Palsy. J Neural Transm (Vienna) 2018;125:1373-9.

20. Süssmuth SD, Uttner I, Landwehrmeyer B, Pinkhardt EH, Brettschneider J, et al. Differential pattern of brain-specific CSF proteins tau and amyloid-beta in Parkinsonian syndromes. Mov Disord 2010;25:1284-8.

21. Mitani K, Furiya Y, Uchihara T, Ishii K, Yamanouchi H, et al. Increased CSF tau protein in corticobasal degeneration. J Neurol 1998;245:44-6.

22. Urakami K, Mori M, Wada K, Kowa H, Takeshima T, et al. A comparison of tau protein in cerebrospinal fluid between corticobasal degeneration and progressive supranuclear palsy. Neurosci Lett 1999;259:127-9. 
23. Urakami K, Wada K, Arai H, Sasaki H, Kanai M, et al. Diagnostic significance of tau protein in cerebrospinal fluid from patients with corticobasal degeneration or progressive supranuclear palsy. J Neurol Sci 2001;183:95-8.

24. Borroni B, Premi E, Agosti C, Alberici A, Cerini C, et al. CSF Alzheimer's disease-like pattern in corticobasal syndrome: evidence for a distinct disorder. J Neurol Neurosurg Psychiatry 2011;82:834-8.

25. Constantinides VC, Paraskevas GP, Efthymiopoulou E, Stefanis L, Kapaki E. Clinical, neuropsychological and imaging characteristics of Alzheimer's disease patients presenting as corticobasal syndrome. J Neurol Sci 2019;398:142-7.

26. Shi M, Bradner J, Hancock AM, Chung KA, Quinn JF, et al. Cerebrospinal fluid biomarkers for Parkinson disease diagnosis and progression. Ann Neurol 2011;69:570-80.

27. Mollenhauer B, Locascio JJ, Schulz-Schaeffer W, Sixel-Doring F, Trenkwalder C, et al. alpha-Synuclein and tau concentrations in cerebrospinal fluid of patients presenting with parkinsonism: a cohort study. Lancet Neurol 2011;10:230-40.

28. Abdo WF, van de Warrenburg BP, Kremer HP, Bloem BR, Verbeek MM. CSF biomarker profiles do not differentiate between the cerebellar and parkinsonian phenotypes of multiple system atrophy. Parkinsonism Relat Disord 2007;13:480-2.

29. Herbert MK, Aerts MB, Beenes M, Norgren N, Esselink RA, et al. CSF neurofilament light chain but not FLT3 ligand discriminates parkinsonian disorders. Front Neurol 2015;6:91.

30. Abdo WF, De Jong D, Hendriks JC, Horstink MW, Kremer BP, et al. Cerebrospinal fluid analysis differentiates multiple system atrophy from Parkinson's disease. Mov Disord 2004;19:571-9.

31. Herbert MK, Eeftens JM, Aerts MB, Esselink RA, Bloem BR, et al. CSF levels of DJ-1 and tau distinguish MSA patients from PD patients and controls. Parkinsonism Relat Disord 2014;20:112-5.

32. Mollenhauer B, Cepek L, Bibl M, Wiltfang J, Schulz-Schaeffer WJ, et al. Tau protein, Abeta42 and S-100B protein in cerebrospinal fluid of patients with dementia with Lewy bodies. Dement Geriatr Cogn Disord 2005;19:164-70.

33. Mollenhauer B, Bibl M, Wiltfang J, Steinacker P, Ciesielczyk B, et al. Total tau protein, phosphorylated tau (181p) protein, betaamyloid(1-42), and beta-amyloid(1-40) in cerebrospinal fluid of patients with dementia with Lewy bodies. Clin Chem Lab Med 2006;44:192-5.

34. Mulugeta E, Londos E, Hansson O, Ballard C, Skogseth R, et al. Cerebrospinal fluid levels of sappalpha and sappbeta in lewy body and Alzheimer's disease: clinical and neurochemical correlates. Int J Alzheimers Dis 2011;2011:495025.

35. Llorens F, Schmitz M, Varges D, Kruse N, Gotzmann N, et al. Cerebrospinal alpha-synuclein in alpha-synuclein aggregation disorders: tau/alpha-synuclein ratio as potential biomarker for dementia with Lewy bodies. J Neurol 2016;263:2271-7.

36. van Steenoven I, van der Flier WM, Scheltens P, Teunissen CE, Lemstra AW. Amyloid-beta peptides in cerebrospinal fluid of patients with dementia with Lewy bodies. Alzheimers Res Ther 2019;11:83.

37. Parnetti L, Tiraboschi P, Lanari A, Peducci M, Padiglioni C, et al. Cerebrospinal fluid biomarkers in Parkinson's disease with dementia and dementia with Lewy bodies. Biol Psychiatry 2008;64:850-5.

38. Kaerst L, Kuhlmann A, Wedekind D, Stoeck K, Lange P, et al. Using cerebrospinal fluid marker profiles in clinical diagnosis of dementia with Lewy bodies, Parkinson's disease, and Alzheimer's disease. J Alzheimers Dis 2014;38:63-73.

39. Gmitterova K, Gawinecka J, Llorens F, Varges D, Valkovic P, et al. Cerebrospinal fluid markers analysis in the differential diagnosis of dementia with Lewy bodies and Parkinson's disease dementia. Eur Arch Psychiatry Clin Neurosci 2018.

40. Gómez-Tortosa E, Gonzalo I, Fanjul S, Sainz MJ, Cantarero S, et al. Cerebrospinal fluid markers in dementia with lewy bodies compared with Alzheimer disease. Arch Neurol 2003;60:1218-22.

41. Boström F, Hansson O, Blennow K, Gerhardsson L, Lundh T, et al. Cerebrospinal fluid total tau is associated with shorter survival in dementia with Lewy bodies. Dement Geriatr Cogn Disord 2009;28:314-9.

42. Abdelnour C, van Steenoven I, Londos E, Blanc F, Auestad B, et al. Alzheimer's disease cerebrospinal fluid biomarkers predict cognitive decline in lewy body dementia. Mov Disord 2016;31:1203-8.

43. Mollenhauer B, Steinacker P, Bahn E, Bibl M, Brechlin P, et al. Serum heart-type fatty acid-binding protein and cerebrospinal fluid tau: marker candidates for dementia with lewy bodies. Neurodegener Dis 2007;4:366-75.

44. Slaets S, Le Bastard N, Theuns J, Sleegers K, Verstraeten A, et al. Amyloid pathology influences abeta1-42 cerebrospinal fluid levels in dementia with lewy bodies. J Alzheimers Dis 2013;35:137-46.

45. van Steenoven I, Aarsland D, Weintraub D, Londos E, Blanc F, et al. Cerebrospinal fluid Alzheimer's disease biomarkers across the spectrum of lewy body diseases: results from a large multicenter cohort. J Alzheimers Dis 2016;54:287-95.

46. Mollenhauer B, Bibl M, Trenkwalder C, Stiens G, Cepek L, et al. Follow-up investigations in cerebrospinal fluid of patients with dementia with Lewy bodies and Alzheimer's disease. J Neural Transm (Vienna) 2005;112:933-48.

47. Bousiges O, Cretin B, Lavaux T, Philippi N, Jung B, et al. Diagnostic value of cerebrospinal fluid biomarkers (Phospho-Tau181, total-Tau, Abeta42, and Abeta40) in prodromal stage of Alzheimer's disease and dementia with lewy bodies. J Alzheimers Dis 2016;51:1069-83.

48. Bousiges O, Bombois S, Schraen S, Wallon D, Quillard MM, et al. Cerebrospinal fluid Alzheimer biomarkers can be useful for discriminating dementia with Lewy bodies from Alzheimer's disease at the prodromal stage. J Neurol Neurosurg Psychiatry 2018;89:467-75.

49. Paraskevas GP, Bougea A, Constantinides VC, Bourbouli M, Petropoulou O, et al. In vivo prevalence of Alzheimer biomarkers in dementia with lewy bodies. Dement Geriatr Cogn Disord 2019;47:289-96.

50. Masliah E, Rockenstein E, Veinbergs I, Sagara Y, Mallory M, et al. beta-amyloid peptides enhance alpha-synuclein accumulation and neuronal deficits in a transgenic mouse model linking Alzheimer's disease and Parkinson's disease. Proc Natl Acad Sci U S A 2001;98:12245-50.

51. Sengupta U, Guerrero-Muñoz MJ, Castillo-Carranza DL, Lasagna-Reeves CA, Gerson JE, et al. Pathological interface between oligomeric alpha-synuclein and tau in synucleinopathies. Biol Psychiatry 2015;78:672-83. 
52. Castillo-Carranza DL, Guerrero-Muñoz MJ, Sengupta U, Gerson JE, Kayed R. $\alpha$-synuclein oligomers induce a unique toxic tau strain. Biol Psychiatry 2018;84:499-508.

53. Borroni B, Gardoni F, Parnetti L, Magno L, Malinverno M, et al. Pattern of Tau forms in CSF is altered in progressive supranuclear palsy. Neurobiol Aging 2009;30:34-40.

54. Borroni B, Malinverno M, Gardoni F, Alberici A, Parnetti L, et al. Tau forms in CSF as a reliable biomarker for progressive supranuclear palsy. Neurology 2008;71:1796-803.

55. Kuiperij HB, Verbeek MM. Diagnosis of progressive supranuclear palsy: can measurement of tau forms help? Neurobiol Aging 2012;33:204.e17-8.

56. Luk C, Compta Y, Magdalinou N, Martí MJ, Hondhamuni G, et al. Development and assessment of sensitive immuno-PCR assays for the quantification of cerebrospinal fluid three- and four-repeat tau isoforms in tauopathies. J Neurochem 2012;123:396-405.

57. Barthélemy NR, Gabelle A, Hirtz C, Fenaille F, Sergeant N, et al. Differential mass spectrometry profiles of tau protein in the cerebrospinal fluid of patients with Alzheimer's disease, progressive supranuclear palsy, and dementia with lewy bodies. J Alzheimers Dis 2016;51:1033-43.

58. Cicognola C, Brinkmalm G, Wahlgren J, Portelius E, Gobom J, et al. Novel tau fragments in cerebrospinal fluid: relation to tangle pathology and cognitive decline in Alzheimer's disease. Acta Neuropathol 2019;137:279-96.

59. Arai H, Morikawa Y, Higuchi M, Matsui T, Clark CM, et al. Cerebrospinal fluid tau levels in neurodegenerative diseases with distinct taurelated pathology. Biochem Biophys Res Commun 1997;236:262-4.

60. Shelley BP, Hodges JR, Kipps CM, Xuereb JH, Bak TH. Is the pathology of corticobasal syndrome predictable in life? Mov Disord 2009;24:1593-9.

61. Ling H, O’Sullivan SS, Holton JL, Revesz T, Massey LA, et al. Does corticobasal degeneration exist? A clinicopathological re-evaluation. Brain 2010;133:2045-57.

62. Lee SE, Rabinovici GD, Mayo MC, Wilson SM, Seeley WW, et al. Clinicopathological correlations in corticobasal degeneration. Ann Neurol 2011;70:327-40.

63. Paik SR, Lee JH, Kim DH, Chang CS, Kim YS. Self-oligomerization of NACP, the precursor protein of the non-amyloid beta/A4 protein (A beta) component of Alzheimer's disease amyloid, observed in the presence of a C-terminal A beta fragment (residues 25-35). FEBS Lett 1998;421:73-6.

64. del Campo M, Mollenhauer B, Bertolotto A, Engelborghs S, Hampel H, et al. Recommendations to standardize preanalytical confounding factors in Alzheimer's and Parkinson's disease cerebrospinal fluid biomarkers: an update. Biomark Med 2012;6:419-30. 